\title{
Personalbemessung in der Langzeitpflege
}

Heinz Rothgang, Mathias Fünfstück und Thomas Kalwitzki

$11.1 \quad$ Einleitung - 148

11.2 Ausgangslage -149

11.3 Chancen und Risiken eines Personalbemessungsverfahrens - 151

11.4 Gesetzlicher Auftrag - 152

11.5 Vorgehensweise - 152

11.5.1 Konzeption - 152

11.5.2 Durchführung - 153

11.6 Erwartete Ergebnisse und Ausblick - 155

Literatur - 156 


\section{- Zusammenfassung}

Die Personalausstattung in deutschen Pflegeheimen wird durchgängig als $z u$ niedrig beschrieben und hat zahlreichen Studien zufolge entsprechende Auswirkungen auf die Versorgungsqualität, den Zustand der Pflegekräfte und deren Verbleib im Beruf. Nach einigen gescheiterten Versuchen bietet der im Zweiten Pflegestärkungsgesetz in $\$ 113 \mathrm{c}$ SGB XI verankerte Gesetzesauftrag nunmehr die Chance auf Einführung eines bundeseinheitlichen Personalbemessungsverfahrens zur Sicherstellung einer Personalmenge und -struktur, die fachgerechte Pflege ohne permanente Überforderung der Pflegekräfte ermöglicht. Der Auftrag zur Entwicklung dieses Instruments ist nach einer europaweiten Ausschreibung an die Universität Bremen gegangen. Im vorliegenden Beitrag werden die dabei genutzte Konzeption und die Durchführung der Studie beschrieben und die erwartbaren Ergebnisse diskutiert.

The number of staff in German nursing homes is considered to be too low and this, according to numerous studies, has a corresponding impact on the quality of care and the health of the nursing staff and on whether they remain in the nursing profession. After a number of failed attempts, the statutory mandate in $\$ 113 c$ SGB XI introduced as part of the Second Long Term Care Strengthening Act provides the opportunity to introduce a national standard procedure for the allocation of staff in order to ensure a workforce size and structure that guarantees professional care without permanently overburdening the nursing staff. After a call for tenders throughout Europe, the University of Bremen was commissioned to develop this instrument. The authors describe the concept used and the implementation of the study and discuss expected results.

\subsection{Einleitung}

In seinem für die Qualitätsdebatte in der gesundheitlichen und pflegerischen Versorgung wegweisendem Artikel hat Donabedian (1966) bereits die Unterscheidung in Struktur-,
Prozess- und Ergebnisqualität vorgenommen. Dabei fördert gute Strukturqualität die Chancen auf gute Prozesse und gute Ergebnisse garantiert sie aber nicht. Wichtigstes Strukturmerkmal in der formellen Langzeitpflege ist sicherlich die Personalausstattung der Einrichtungen - in quantitativer und qualitativer Hinsicht.

Schon bei Einführung der Pflegeversicherung wurde daher die Personalbemessung in Pflegeheimen diskutiert, aber weniger in Bezug auf die Pflegequalität als vielmehr auf die Belastung der Pflegekräfte auf der einen Seite (vgl. den Überblick bei Zimber 1998) und die Pflegekosten auf der anderen Seite. Mit dem Standard-Pflegesatz-Modell (SPM) der Spitzenverbände der Pflegekassen, der Bundesarbeitsgemeinschaft der überörtlichen Träger der Sozialhilfe und der Bundesvereinigung der kommunalen Spitzenverbände (siehe z. B. Moldenhauer und Fink 1997) wurde ein Vorstoß zur Vereinheitlichung der Personalausstattung und Pflegesätze unternommen, der nach Ansicht der Einrichtungsträger aber zu deutlichen Kürzungen der Personalausstattungen geführt hätte (Winkler 2000). Angesichts der massiven Kritik wurde das SPM von den Initiatoren zurückgezogen und „offiziell verworfen“ (Strünck 1998, S. 145). Empirisch ließ sich jedoch beispielsweise in Nordrhein-Westfalen eine Angleichung der Pflegesätze zumindest innerhalb eines Bundeslandes beobachten. Anders als von den Gegnern des SPM befürchtet jedoch nicht nach unten, sondern nach oben (Roth und Rothgang 1999, 2000).

Die normative Frage nach einer angemessenen Personalausstattung wurde vor rund zwanzig Jahren dann erstmals ernsthaft angegangen, als das Kuratorium Deutsche Altershilfe (KDA) beauftragt wurde, die Übertragbarkeit des in Kanada entwickelten PLAISIR-Verfahrens auf Deutschland zu überprüfen. Obgleich der Auftragnehmer die Anwendbarkeit im deutschen Kontext im Rahmen einer Erprobung in elf Einrichtungen der Arbeiterwohlfahrt bestätigte (KDA 2002) und auch der Überprüfungsprozess des Verfahrens zur Anwendung auf Landesebene - einschließlich des Transfers der 
Instrumente aus dem kanadischen in den deutschen Kontext - nach Ansicht des Auftragnehmers gelungen ist (KDA 2003), wurde PLAISIR letztlich nicht in Deutschland eingeführt. Hierfür wurden zwei Gründe genannt: Zum einen wurde auf Lizenzschwierigkeiten mit dem kanadischen Rechteinhaber verwiesen und zum anderen wurde bemängelt, dass das System eine „Blackbox“ und es aus lizenzrechtlichen Gründen nicht möglich sei, die dahinter liegenden Algorithmen näher anzuschauen. Welche weiteren Interessen bei der Ablehnung letztlich noch eine Rolle gespielt haben, kann hier nicht weiter diskutiert werden. Eine Lehre, die aus dem Scheitern von PLAISIR gezogen werden kann, ist aber, dass neuerliche Versuche zur Einführung eines Personalbemessungsverfahrens sollten auf offenen Algorithmen beruhen, die in direkter Verbindung zu den Rahmenbedingungen in Deutschland entwickelt wurden und bei denen Lizenzprobleme von vornherein ausgeschlossen sind.

Mit dem Scheitern von PLAISIR sind die Forderungen nach Einführung eines Personalbemessungsverfahrens aber nicht verstummt. So hat der GKV-Spitzenverband im Rahmen des Modellprogramms nach $₫ 8$ Abs. 3 SGB XI ein Modellprojekt zur „Entwicklung und Erprobung von Grundlagen der Personalbemessung in vollstationären Pflegeeinrichtungen auf der Basis des Bedarfsklassifikationssystems der Referenzmodelle" gefördert, dessen Abschlussbericht 2010 vorgelegt wurde (Wingenfeld et al. 2010). Auch seitens der Gewerkschaften wurde ein Personalbemessungsverfahren nach wie vor thematisiert, u. a. durch Beauftragung eines Gutachtens zur „Gesetzlichen Personalbemessung in der stationären Altenpflege" (Greß und Stegmüller 2016).

Tatsächlich hat der Gesetzgeber im Zweiten Pflegestärkungsgesetz einen erneuten Anlauf genommen und die gemeinsame Selbstverwaltung in der Pflege beauftragt, bis Juni 2020 ein Personalbemessungsverfahren zu entwickeln und $\mathrm{zu}$ erproben und sich dabei wissenschaftlichen Sachverstands zu bedienen. Der entsprechende Entwicklungsauftrag ist europaweit ausgeschrieben und schließlich an die Universität Bremen vergeben worden. Nachfol- gend wird berichtet, wie bei seiner Umsetzung vorgegangen wird ( $\triangleright$ Abschn. 11.5) und welche Ergebnisse und daraus resultierenden Anforderungen an die Weiterentwicklung von Einrichtungen zu erwarten sind ( $\triangleright$ Abschn. 11.6). Zuvor werden aber noch einmal kurz die Ausgangslage skizziert ( Abschn. 11.2), die Chancen und Risiken eines Personalbemessungsverfahrens reflektiert ( Abschn. 11.3) und der Gesetzesauftrag umschrieben (> Abschn. 11.4).

\subsection{Ausgangslage}

Die Personalausstattung in deutschen Pflegeheimen wird inzwischen - ebenso wie die in deutschen Krankenhäusern - durchgängig als $z u$ niedrig beschrieben. Pflegekräfte fühlen sich gehetzt und leiden unter den Folgen einer Arbeitsintensivierung (DGB 2018, S. 7 f.). Etwa die Hälfte der befragten Pflegekräfte gibt dabei an, ihr (verdichtetes) Arbeitspensum nur bewältigen zu können, indem sie kompensatorisch Abstriche bei der Qualität ihrer Dienstleistung macht (ebd., S. 16 f.). Eine fachgerechte Erbringung der Pflege muss somit systematisch als gefährdet gelten. Gesundheitsbezogene Belastungen führen zu erhöhter Krankheitsdauer und vermehrten Krankheitstagen der Pflegenden (Isfort et al. 2018, S. 2 f.), wodurch wiederum die Arbeitsverdichtung verstärkt wird. Solche Arbeitsbedingungen sind auch der Hauptgrund für den vorzeitigen Ausstieg von Pflegekräften aus dem Beruf (Hasselhorn et al. 2005), durch den der Pflegenotstand, also die Schwierigkeiten, vorhandene Stellen zu besetzen, weiter verstärkt wird. So nannten die im „Pflexit-Monitor“ des Medizinprodukteherstellers Paul Hartmann befragten Pflegekräfte den „permanenten Personalmangel“ (72\%) sowie die "generell hohe Arbeitsbelastung“ (57\%) als Hauptgründe für ihre berufliche Unzufriedenheit (Ärztezeitung vom 16.03.2018).

Um die in Zukunft steigende Zahl Pflegebedürftiger in Pflegeheimen angemessen versorgen zu können, muss daher die Zahl der Beschäftigten pro Pflegebedürftigen erhöht werden. Das setzt die Ausweitung der Zahl der 
Pflegestellen voraus. Gerade diese Ausweitung trüge dann auch zur Steigerung der Attraktivität des Pflegeberufs bei, die notwendig ist, um (auch) die neugeschaffenen Stellen überhaupt besetzen zu können. Um das Ausmaß der notwendigen Personalbesetzung bestimmen zu können, ist aber die Entwicklung valider Verfahren zur Bestimmung der notwendigen Personalmengen erforderlich.

Gleichzeitig ist die aktuelle Situation durch erhebliche regionale Ungleichheiten gekennzeichnet. Die Personalbesetzung in deutschen Pflegeheimen wird dabei entscheidend durch die sogenannten Stellenschlüssel geprägt, die in den Rahmenverträgen gemäß $₫ 75$ SGB XI auf Länderebene beschlossen werden. Darin werden für die einzelnen Pflegestufen - inzwischen Pflegegrade - Verhältniszahlen von Vollzeitpflegekraft zu Bewohnern festgelegt, die zum Teil auch in Form von Korridoren angegeben werden. Um eine Vergleichbarkeit zu ermöglichen, ist in - Tab. 11.1 auf Bundesund Landesebene angegeben, wie viele Pflegekräfte (gemessen in Vollzeitäquivalenten) für eine Einrichtung mit 100 Bewohnern vorgesehen sind, deren Pflegestufenverteilung dem Bundes- und Landesdurchschnitt entspricht. Insoweit die Landesregelungen Korridore vorsehen, wurde dabei in der Regel auf den Mittelwert der Korridore abgestellt (vgl. Rothgang und Wagner 2019 für Details der Berechnung).

Wie die Tabelle zeigt, unterscheiden sich die Bundesländer erheblich. Während in Bayern zur Versorgung der 100 Bewohner mehr als 40 (Vollzeit)Pflegekräfte vorgesehen sind, sind es in Mecklenburg-Vorpommern, im Saarland oder in Sachsen-Anhalt weniger als 35. Zur Verdeutlichung dieser Unterschiede ist in - Abb. 11.1 angegeben, um wieviel die Zahl der von einer Pflegekraft zu versorgenden Bewohner in den einzelnen Bundesländern den bayerischen Wert überschreitet, der 2016 die höchste Personalintensität aufgewiesen hat. In zehn Bundesländern übersteigt die Zahl der Bewohner, die pro Pflegekraft versorgt werden muss, den bayerischen Wert um mehr als $10 \%$, davon in vier Ländern sogar um mehr als $15 \%$.
- Tabelle 11.1 Pflegekräfte für eine Einrichtung mit 100 Bewohnern bei bundes- bzw. landesdurchschnittlicher Pflegestufenstruktur gemäß Personalschlüssel Mitte 2016

\begin{tabular}{|l|l|}
\hline Bundesland & Anzahl Pflegekräfte \\
\hline Baden-Württemberg & 39,53 \\
\hline Bayern & 40,46 \\
\hline Berlin & 36,06 \\
\hline Brandenburg & 35,49 \\
\hline Bremen & 36,22 \\
\hline Hamburg & 37,33 \\
\hline Hessen & 38,49 \\
\hline Mecklenburg-Vorpommern & 34,66 \\
\hline Niedersachsen & 35,05 \\
\hline Nordrhein-Westfalen & 36,69 \\
\hline Rheinland-Pfalz & 36,05 \\
\hline Saarland & 34,84 \\
\hline Sachsen & 39,24 \\
\hline Sachsen-Anhalt & 34,46 \\
\hline Schleswig-Holstein & 38,07 \\
\hline Thüringen & 35,34 \\
\hline Mittelwert & 36,75 \\
\hline Bund & 37,42 \\
\hline
\end{tabular}

Quelle: Rothgang und Wagner 2019

Pflege-Report 2019

Da es sich bei der Pflegeversicherung um eine bundesweite Sozialversicherung handelt und auch die Einstufung der Heimbewohner nach bundeseinheitlichen Maßstäben erfolgt, ist es aber schwer begründbar, warum sich die Personalmenge, die für die Versorgung eines Heimbewohners aufgebracht wird, zwischen den Ländern derart unterscheidet. Ein bundesweites Personalbemessungsverfahren kann hier zu einer Angleichung führen, die im Hinblick auf das Verfassungsgebot der Gleichartigkeit der Lebensverhältnisse im Bundesgebiet als geboten erscheint. 


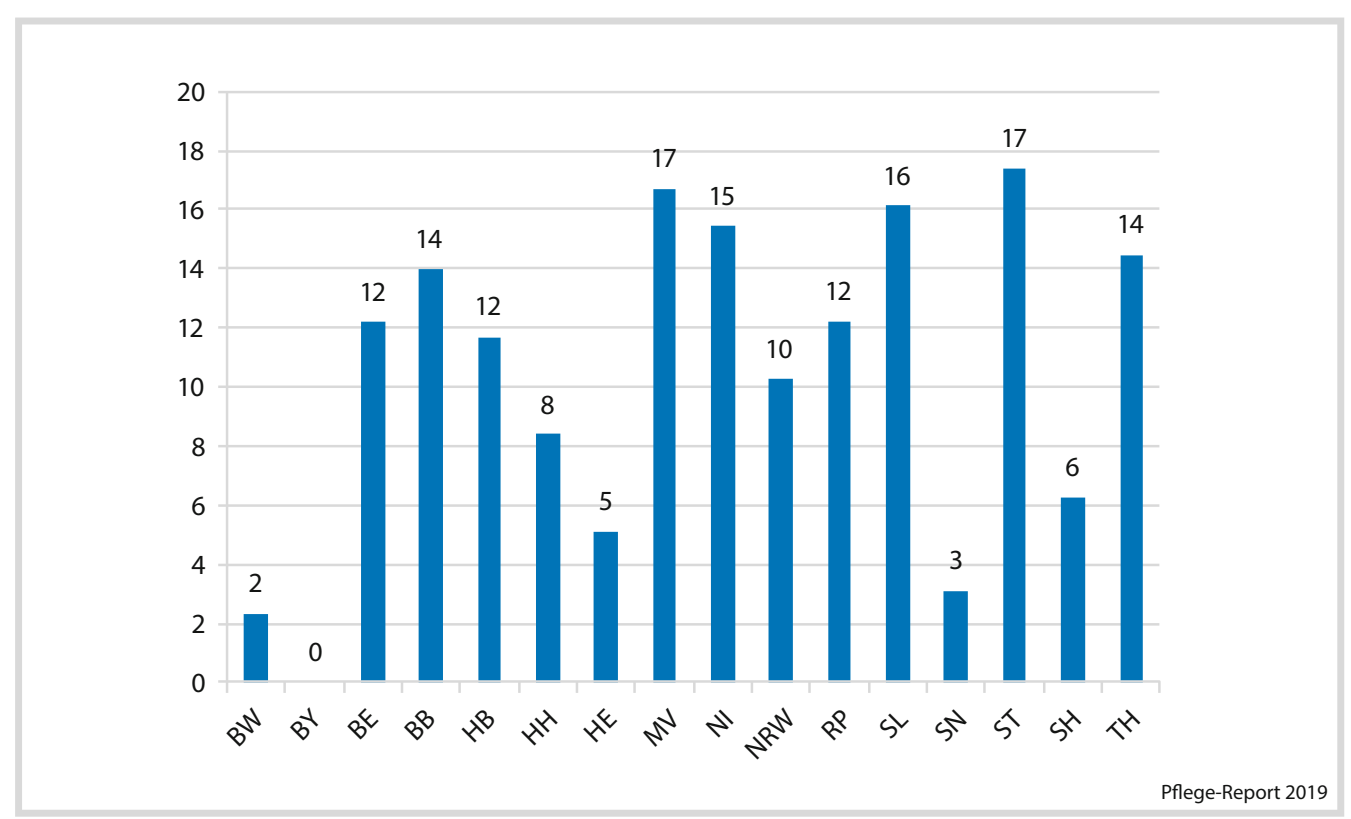

- Abb. 11.1 Im Vergleich zu Bayern zusätzlich zu versorgende Heimbewohner pro Pflegekraft gemäß Personalschlüsseln und landesdurchschnittlichem Pflegestufenmix, in \%. (Quelle: eigene Berechnungen basierend auf den Werten aus $\bullet$ Tab. 11.1)

\subsection{Chancen und Risiken eines Personalbemessungs- verfahrens}

Ein bundesweites Personalbemessungsverfahren bietet die Chance zur Sicherstellung einer Personalmenge in Einrichtungen, die ausreicht, um fachgerechte Pflege ohne permanente Überforderung der Pflegekräfte zu ermöglichen. Gleichzeitig kann es zu einem Abbau der fachlich nicht erklärbaren und normativ nur schwer begründbaren regionalen Unterschiede beitragen und damit die beiden in Abschn. 11.2 genannten Hauptprobleme der Ausgangslage adressieren.

Neben diesen Chancen birgt die Einführung eines bundesweiten Personalbemessungsverfahrens aber auch Risiken. Personalbemessungsverfahren können zu einer Verkrustung der Strukturen führen und Innovationen verhindern, wenn seitens der Kostenträger in Preisverhandlungen die Beteiligten nur noch prüfen, ob die Personalzahlen eingehalten werden und die Anbieter - ohne Berücksichtigung der Einrichtungsspezifika - ihr Personaltableau ausschließlich an den Ergebnissen des Personalbemessungsverfahrens orientieren. Entsprechende Erfahrungen wurden in Deutschland in den 1970er und 1980er Jahren im Krankenhausbereich gemacht, als die Personalanhaltszahlen der Deutschen Krankenhausgesellschaft aus dem Jahre 1969 in Pflegesatzverhandlungen zur alleinigen Entscheidungsgröße wurden und innovative Personalmixe effektiv verhindert haben. Um dies zu vermeiden, müssen die Ergebnisse des Personalbemessungsverfahrens im Pflegebereich als Grundlage einrichtungsindividueller Verhandlungen angesehen werden, nicht aber als deren vorweggenommenes Ergebnis.

Problematisch kann auch der Übergangsprozess sein. Sollten die Ergebnisse des Personalbemessungsverfahrens und der sich anschließenden Verhandlungen weit von den bisherigen Personalausstattungen entfernt sein, 
muss den Einrichtungen hinreichend Zeit gegeben werden, den Anpassungsprozess zu organisieren. Eine Konvergenzphase, wie es sie nach der Einführung der DRG-basierten Fallpauschalenvergütung im deutschen Krankenhauswesen von 2003 bis 2008 gegeben hat, wäre hier zielführend.

\subsection{Gesetzlicher Auftrag}

Gemäß dem im Rahmen des Zweiten Pflegestärkungsgesetzes neu in das SGB XI eingeführten $\$ 113 \mathrm{c}$ haben die Vertragsparteien nach $\$ 113$ SGB XI die Entwicklung und Erprobung eines wissenschaftlich fundierten Verfahrens zur einheitlichen Bemessung des Personalbedarfs in Pflegeeinrichtungen für direkte und indirekte pflegerische Maßnahmen sowie für Hilfen bei der Haushaltsführung nach qualitativen und quantitativen Maßstäben sicherzustellen. Die Vertragsparteien erarbeiten das Verfahren nicht selbst, sondern „beauftragen zur Sicherstellung der Wissenschaftlichkeit des Verfahrens fachlich unabhängige wissenschaftliche Einrichtungen" ( $\$ 113 c$ Abs. 1 Satz 5 SGB XI). Die Entwicklung und Erprobung des Verfahrens hat bis zum 30. Juni $2020 \mathrm{zu}$ erfolgen.

Diese Norm ist in mehrfacher Perspektive bemerkenswert. Zunächst wird hier ein „Verfahren zur einheitlichen Bemessung " gefordert, nicht aber bundeseinheitliche Personalziffern. Damit bleibt die Möglichkeit offen, im Rahmen des einheitlichen Verfahrens Länderspezifika zu berücksichtigen. Gleichzeitig wirkt die „Bundeseinheitlichkeit“ jedoch auf eine Verringerung der Differenzen zwischen den Ländern hin. Der Gesetzesauftrag bezieht sich dabei nur auf die Entwicklung und Erprobung - nicht aber auf die Einführung. Damit ist klar, dass über die Einführung eines bundeseinheitlichen Personalbemessungsverfahrens erst nach Vorlage des Verfahrens selbst entschieden werden wird.

Der Bundesgesetzgeber kann versuchen, ein solches Verfahren sozialrechtlich im Recht der Pflegeversicherung zu verankern oder eine ordnungsrechtliche Einführung durch die
Länder zu betreiben. Auch eine sozialrechtliche Einführung wird aber der Zustimmung des Bundesrats bedürfen, sodass die Länder auf alle Fälle eine zentrale Bedeutung für die Einführung eines solchen Verfahrens haben. Allerdings hat schon die Einführung des neuen Pflegebedürftigkeitsbegriffs gezeigt, dass derartige Innovationen nur dann umgesetzt werden, wenn die Akteure im Feld deren Notwendigkeit nachdrücklich thematisieren.

Nach europaweiter Ausschreibung ist der Auftrag an die Universität Bremen ergangen. In Umsetzung dieses Auftrags hat es sich das Projektteam zum Ziel gesetzt, ein Personalbemessungsverfahren zu entwickeln, das auf Basis der Anzahl versorgter Pflegebedürftiger und dem Ausmaß ihrer Pflegebedürftigkeit, wie sie im Rahmen der Begutachtung mit dem Begutachtungsinstrument erhoben wird, nach Qualifikationsstufen differenzierte Personalmengen errechnet, die dann Grundlage für landesspezifische Setzungen und einrichtungsbezogene Verhandlungen sein können.

\subsection{Vorgehensweise}

Bei der Beschreibung der Vorgehensweise wird nachfolgend zunächst auf die Konzeption ( Abschn. 11.5.1) und dann auf die Durchführung ( $\triangleright$ Abschn. 11.5.2) eingegangen.

\subsubsection{Konzeption}

Die Entwicklung eines bundeseinheitlichen Bemessungsverfahrens zielt zum einen darauf, fachlich nicht erklärbare (regionale) Unterschiede abzubauen. Hierbei geht es um Verteilungsgerechtigkeit, aber noch nicht um Bedarfsgerechtigkeit. Um diesem Ziel gerecht $\mathrm{zu}$ werden, bietet sich ein empirischer Ansatz an, der die Unterschiede zwischen Einrichtungen erfasst und Durchschnitte als Orientierungswerte ermittelt, von denen nur abgewichen werden darf, wenn dies durch die Einrichtungskonzeption fachlich begründet werden kann. 
Zugleich geht es aber auch darum, eine "gute" Strukturqualität durch fachlich angemessene Personalmengen und -strukturen in Bezug auf Art und Ausmaß der Pflegebedürftigkeit der jeweiligen Bewohner sicherzustellen. Dieses Postulat zielt auf Bedarfsgerechtigkeit ab und erzwingt einen analytischen Zugang, bei dem festgelegt wird, welche Personalmengen in welchem Qualifikationsmix für eine fachgerechte Leistungserbringung notwendig sind. Hierzu ist eine fachliche und politische Konsentierung notwendig, da es sich hierbei um sozial konstruierte normative Setzungen handelt, die sich nur als Ergebnis einer Übereinkunft der Beteiligten ergeben können.

Um SOLL-Zahlen für eine bedarfsgerechte Personalbemessung $\mathrm{zu}$ ermitteln, werden in der gewählten Vorgehensweise ein empirischer und ein analytischer Ansatz miteinander verknüpft. Hierzu werden die drei Dimensionen der Leistungserbringung betrachtet: die Zahl der bedarfsnotwendigen Interventionen pro Bewohner, die bedarfsgerechte Zeit pro Intervention für den entsprechenden Bewohner und das bedarfsgerechte Qualifikationsniveau der leistungserbringenden Person für diese Intervention. Die Projektkonzeption sieht dabei vor, jeweils das IST zu messen, simultan zu prüfen, inwieweit für eine bedarfsgerechte Versorgung hierbei $\mathrm{Zu}$ - oder Abschläge (bei Menge, Zeit und/oder Qualifikationsniveau) notwendig gewesen wären. Diese $\mathrm{Zu}$ - und Abschläge werden als Delta bezeichnet und bilden in Summe mit dem IST der Erbringung die jeweiligen fachgerechten SOLL-Werte.

Bei der Umsetzung dieser Konzeption wurde im ersten Schritt auf Basis der vorhandenen (Lehrbuch)Literatur ein sogenannter Interventionskatalog entwickelt, der es erlaubt, alle im Pflegeheim erbrachten Leistungen zu erfassen. Bei der Entwicklung dieses Katalogs wurden die Anforderungen des neuen Pflegebedürftigkeitsbegriffs und die daraus resultierenden Implikationen für einen korrespondierenden Pflegebegriff (vgl. Wingenfeld und Büscher 2017) berücksichtigt. Für alle Interventionen wurden dann - in Abhängigkeit von der mittels des Begutachtungsinstruments abgebilde- ten Art und dem Ausmaß der Pflegebedürftigkeit - fachlich gesetzte Teilschritte und Anforderungen sowie Qualifikationsanforderungen (QN-Anforderungen) festgelegt, die im Handbuch zum Interventionskatalog sowie dem $\mathrm{Ka}$ talog der QN-Anforderungen normiert wurden. Diese Instrumente wurden fachlich und politisch konsentiert. Sie erlauben es, in Erhebungen (s. > Abschn. 11.5.2) nicht nur die aktuelle Situation im IST zu erfassen, sondern sie darüber hinaus fachlich zu bewerten und durch $\mathrm{Zu}$ und Abschläge auf das IST die SOLL-Werte zu berechnen (•Abb. 11.2).

\subsubsection{Durchführung}

Hierzu wurden Daten in 62 vollstationären Erhebungseinheiten unter der Beteiligung von insgesamt 1.380 Bewohnern erfasst. Die Datenerhebung umfasste dabei - nach der Rekrutierungsphase, in der Bewohnerinnen und Bewohner für eine Studienteilnahme angeworben wurden - in jeder Einrichtung einen Zeitraum von mindestens vier Wochen (• Abb. 11.3). Um Art und Ausmaß der Pflegebedürftigkeit der betroffenen Bewohner zu erfassen, wurde von Gutachtern der MDK-Gemeinschaft und der Medicproof GmbH für jeden Studienteilnehmer ein aktuelles Assessment mittels des Begutachtungsinstruments durchgeführt (2. Woche). Basierend auf diesen Informationen, der individuellen Pflegedokumentation und - bei Bedarf - einer Inaugenscheinnahme des Bewohners wurde sodann von wissenschaftlichen Mitarbeiterinnen und Mitarbeitern des Studienteams gemeinsam mit einer zu diesem Zweck freigestellten Bezugspflegekraft der Einrichtung eine Pflegeplanung erstellt, die die bedarfsnotwendigen Interventionen tagesstrukturiert abbildet (3. Woche). Diese Planung wurde in der Erhebung als Erfassungsraster genutzt und zu diesem Zweck auf Tablet-Computer übertragen, die den Datenerhebern zur Verfügung gestellt wurden. Ausgerüstet mit diesen Tablets haben insgesamt 242 datenerhebende Pflegefachkräfte über jeweils fünf Tage alle in den 


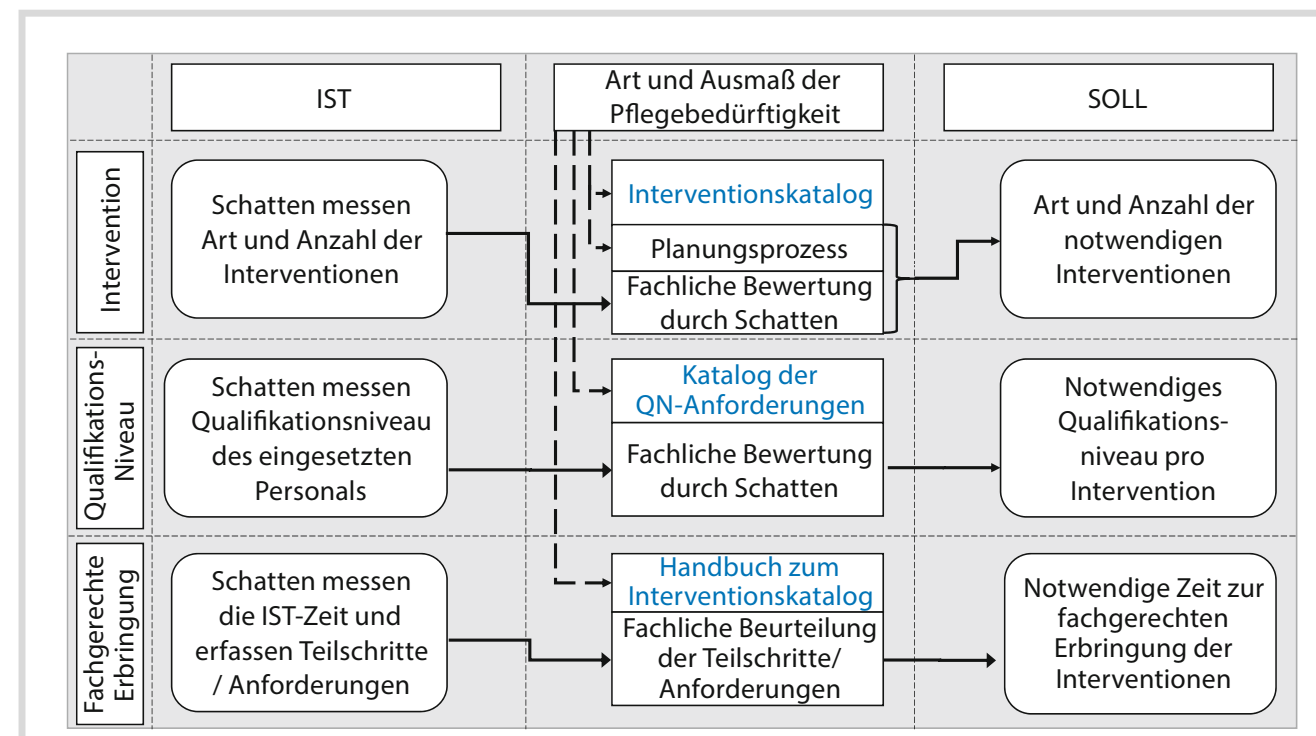

Pflege-Report 2019

- Abb. 11.2 Schematische Darstellung der Grundkonzeption der Studie

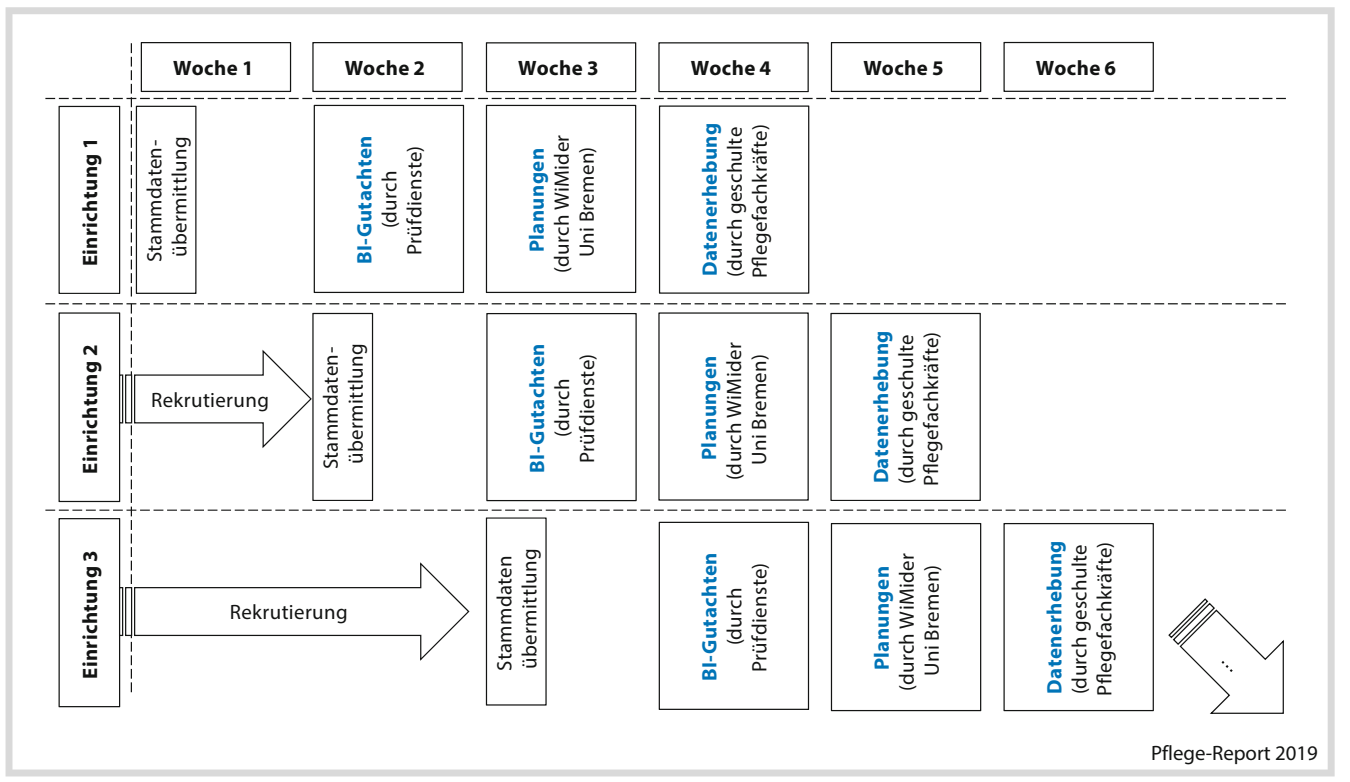

- Abb. 11.3 Schematische Darstellung der Projektdurchführung

Erhebungseinheiten eingesetzten Pflegekräfte "beschattet" (4. Woche). Die Schatten wurden dabei etwa zur Hälfte von der MDKGemeinschaft und dem Verband der privaten Krankenversicherung (PKV) einerseits und von den Einrichtungsträgern (privaten, freigemeinnützigen und kommunalen, jeweils etwa gemäß ihrem Anteil an den Pflegeeinrichtungen) andererseits rekrutiert und in gemischten Teams eingesetzt. 
Nach einer zweitägigen Schulung, in der die Handhabung der Instrumente und Tablets sowie das in den Teilschritten und Anforderungen zum Ausdruck kommende Pflegeverständnis vermittelt wurden, haben die Schatten jeweils für eine ganze Schicht eine Pflegekraft eins zu eins begleitet. Dabei erfassten sie nicht nur die Erbringung der Pflege sekundengenau, sondern bewerteten gleichzeitig anhand der Teilschritte und Anforderungen, die für jede Intervention bei einem Bewohner individualisiert auf dem Tablet vorlagen, die Notwendigkeit und die fachgerechte Durchführung jeder einzelnen Intervention. Wurde dabei festgestellt, dass notwendige Teilschritte ausgelassen oder Anforderungen nicht beachtet wurden, vergaben die Schatten Zeitzuschläge. Analog hierzu wurden zeitliche Abschläge vergeben, wenn eine Intervention ineffizient erfolgte oder einzelne unnötige Teilschritte erbracht wurden. Ebenso wurden bedarfsnotwendige, aber nicht erbrachte Interventionen als solche erfasst. Umgekehrt konnten erbrachte Interventionen als nicht notwendig gekennzeichnet werden, sodass sie bei der Auswertung aus der Menge der erforderlichen Leistungen ausgeschlossen werden konnten. Im Rahmen eines rollierenden Systems wurden im Zeitraum von April bis Oktober 2018 alle teilnehmenden Einrichtungen besucht. Insgesamt wurden in vollstationären Einrichtungen in 2.046 beschatteten Schichten Daten zu mehr als 144.000 Interventionen erfasst, die ausgewertet werden konnten.

\subsection{Erwartete Ergebnisse und Ausblick}

Der als Ergebnis der Studie zu entwickelnde Algorithmus produziert für jede Einrichtung eine nach Qualifikationsgraden gegliederte Personalmenge, die zur fachgerechten Pflege erforderlich ist. Zahl und Qualifikationsstruktur der Pflegekräfte hängen dabei von der Zusammensetzung der Bewohnerschaft ab. Dadurch wird eine einrichtungsübergreifende Fachkraftquote durch einrichtungsindividuell bedarfsnotwendi- ge Personalmixe ersetzt. Erste Ergebnisse zeigen, dass deutlich mehr Assistenzkräfte, aber nur in geringem Umfang zusätzliche Fachkräfte benötigt werden. Ein derart veränderter Mix weist bereits darauf hin, dass die Rolle von Fachkräften in Einrichtungen weiterentwickelt werden muss: Diese müssen deutlich stärker in Planung, Koordination und Anleitung eingesetzt werden, während risikoarme pflegerische Aufgaben stärker an Assistenzkräfte, die gezielt weiterzubilden sind, übertragen werden müssen.

Dementsprechend sollte die Einführung des Personalbemessungsverfahrens durch Personal- und Organisationsentwicklung flankiert werden, da nur so sichergestellt werden kann, dass resultierende Personalsteigerungen sinnvoll zur Steigerung der Pflegequalität und Reduktion der Arbeitsbelastung in der Pflege verwendet werden. Schon aus diesem Grund kann ein Personalbemessungsinstrument, das verbindliche Vorgaben für die Personalmengen macht, nicht von einem Tag auf den anderen eingeführt werden.

Zudem können höhere Personalmengen nur durch die Einstellung von Personal umgesetzt werden, wenn auch die Ausbildungskapazitäten erhöht und letztlich mehr Pflegekräfte ausgebildet werden. Derartige Maßnahmen greifen aber erst nach einer gewissen Zeit. Denkbar ist daher - analog zur Einführung einer DRG-basierten Fallpauschalenfinanzierung im Krankenhausbereich von 2003 bis 2008 - ein schrittweiser Personalaufbau mit festen jährlichen Steigerungsraten.

Folglich ist es notwendig, eine Roadmap für die Umsetzung eines Personalbemessungsverfahrens $\mathrm{zu}$ entwickeln. Diese sollte nach Abschluss der Entwicklung und formalen Erprobung des Instruments in einigen Einrichtungen pilotiert werden. Dabei wäre eine Personalstruktur dem Instrument entsprechend einzusetzen und zu testen, mit welchen organisatorischen Entwicklungen erreicht werden kann, dass die quantitative Personalveränderung auch zu einer qualitativen Verbesserung der Pflegepraxis führt. In diesem Rahmen kann auch geprüft werden, ob diese organisatori- 
schen Weiterentwicklungen - verknüpft mit einer zunehmenden Digitalisierung - auch schon vor Erreichen der letzten Ausbaustufe der Personalsteigerung zu einer auskömmlichen Personalsituation führt. Der sofortige Beginn des Stufenplans mit einem definierten Ausbauziel würde dabei die Attraktivität des Berufs erhöhen und die Rekrutierung von Pflegekräften unmittelbar erleichtern. Die parallele Weiterentwicklung der Heimstrukturen kann zeigen, inwieweit der durch den Stellenzuwachs - insbesondere bei den Assistenzkräften - entstehende neue Personalmix bereits zu besseren Ergebnissen führt, sodass das Instrument nachjustiert werden kann. Nach Ablauf der Konvergenzphase wäre dann eine Personalausstattung erreicht, die fachgerechte Pflege ermöglicht. Individuelle Anpassungen der rechnerisch resultierenden Personalmengen aufgrund spezifischer Einrichtungskonzeptionen könnten im Rahmen der Pflegesatzverhandlungen weiterhin vereinbart werden.

\section{Literatur}

DGB - Deutscher Gewerkschaftsbund (2018) Arbeitsbedingungen in der Alten- und Krankenpflege. So beurteilen die Beschäftigten die Lage. Ergebnisse einer Sonderauswertung der Repräsentativumfragen zum DGB-Index Gute Arbeit. https://index-gutearbeit.dgb.de/++co++df07ee92-b1 ba-11e8-b39252540088cada. Zugegriffen: 9. Apr. 2019

Donabedian A (1966) Evaluating the quality of medical care. Milbank Mem Fund Q 44(3):166-203

Greß S, Stegmüller K (2016) Gesetzliche Personalbemessung in der stationären Altenpflege. Gutachterliche Stellungnahme für die Vereinte Dienstleistungsgewerkschaft (ver.di). pg-papers (Diskussionspapiere aus dem Fachbereich Pflege und Gesundheit der Hochschule Fulda) Nr. 01/2016. https://www. verdi.de/++file++56cd87e7bdf98d086200021a/ download/Gutachten_gress_stegmueller.pdf. Zugegriffen: 9. Apr. 2019

Hasselhorn H-M, Müller BH, Tackenberg P (2005) Die Untersuchung des vorzeitigen Ausstiegs aus dem Pflegeberuf in Europa - die europäische NEXT-Studie. In: Hasselhorn H-M, Müller BH, Tackenberg P, Kümmerling A, Simon M (Hrsg) Berufsausstieg bei Pflegepersonal. Arbeitsbedingungen und beabsichtigter Berufsausstieg bei Pflegepersonal in Deutschland und Europa. Wirtschaftsverlag, Bremerhaven, S 11-20
Isfort M, Rottländer R, Weidner F, Gehlen D, Hylla J, Tucman D (2018) Pflege-Thermometer 2018. Eine bundesweite Befragung von Leitungskräften zur Situation der Pflege und Patientenversorgung in der stationären Langzeitpflege in Deutschland

KDA - KDA Beratungs- und Forschungsgesellschaft für Altenhilfe mbH (2002) Qualitative und quantitative Erfassung des erforderlichen Pflegezeit- und Personalbedarfs in deutschen Altenpflegeheimen. Erprobung des Verfahrens PLAISIR in elf Einrichtungen der Arbeiterwohlfahrt. Band 225 der Schriftenreihe des Bundesministeriums für Familie, Senioren, Frauen und Jugend. Kohlhammer, Stuttgart

KDA - KDA Beratungs- und Forschungsgesellschaft für Altenhilfe mbH (2003) Analyse und Transfer des Verfahrens PLAISIR $\odot$. Vorbereitung und Dokumentation der Überprüfungsprozesse des Verfahrens PLAISIR@ zur Anwendung auf Landesebene. KDA, Köln

Moldenhauer M, Fink G (1997) Standard-Pflegesätze für die vollstationäre Pflege. Die Betriebskrankenkasse 3:124-132

Roth G, Rothgang H (1999) Die Auswirkungen des Pflege-Versicherungsgesetzes auf die Entwicklung der Heimentgelte. Zeitschrift Für Gesundheitswissenschaften 4:307-336

Roth G, Rothgang H (2000) „Angleichung nach oben“: Die Entwicklung der Heimentgelte nach Einführung der Pflegeversicherung. Nachrichtendienst des Deutschen Vereins für öffentliche und private Fürsorge 3:85-90

Rothgang H, Wagner C (2019) Quantifizierung der Personalverbesserungen in der stationären Pflege im Zusammenhang mit der Umsetzung des Zweiten Pflegestärkungsgesetzes. Expertise für das Bundesministerium für Gesundheit. https://www.bundesgesundheitsministerium. de/fileadmin/Dateien/5_Publikationen/Pflege/ Berichte/Abschlussbericht_Quantifizierung_der_ Personalverbesserungen.pdf. Zugegriffen: 9. Apr. 2019

Strünck C (1998) Pflegeversicherung - Barmherzigkeit mit beschränkter Haftung. Institutioneller Wandel, Machtbeziehungen und organisatorische Anpassungsprozesse. Leske + Budrich, Opladen

Wingenfeld K, Büscher A (2017) Strukturierung und Beschreibung pflegerischer Aufgaben auf der Grundlage des neuen Pflegebedürftigkeitsbegriffs. Unter Mitarbeit von D. Wibbeke. Universität Bielefeld, Institut für Pflegewissenschaft (Hrsg). Bielefeld/Osnabrück. https://www. bundesgesundheitsministerium.de/fileadmin/ Dateien/5_Publikationen/Pflege/Berichte/ Fachbericht_Pflege.pdf. Zugegriffen: 6. März 2019

Wingenfeld K, Ammann A, Ostendorf A (2010) Abschlussbericht der wissenschaftlichen Begleitung zum Modellprojekt: „Entwicklung und Erprobung von Grundlagen der Personalbemessung in voll- 
stationären Pflegeeinrichtungen auf der Basis des Bedarfsklassifikationssystems der Referenzmodelle". https://www.gkv-spitzenverband.de/ pflegeversicherung/forschung/modellprojekte/ pflege_abgeschlossene_projekte_8/entwicklung_ erprobung.jsp. Zugegriffen: 9. Apr. 2019

Winkler T (2000) Leistungsgerechte Pflegesätze im Bereich „Stationärer Altenhilfe" nach dem Pflegever- sicherungsgesetz - Eine kritische Auseinandersetzung mit dem "Standard-Pflegesatz-Modell“. Pflege Ges 5(4):95-100

Zimber A (1998) Beanspruchung und Streß in der Altenpflege: Forschungsstand und Forschungsperspektiven. Z Gerontol Geriatr 31:417-425

Open Access Dieses Kapitel wird unter der Creative Commons Namensnennung 4.0 International Lizenz (http:// creativecommons.org/licenses/by/4.0/deed.de) veröffentlicht, welche die Nutzung, Vervielfältigung, Bearbeitung, Verbreitung und Wiedergabe in jeglichem Medium und Format erlaubt, sofern Sie den/die ursprünglichen Autor(en) und die Quelle ordnungsgemäß nennen, einen Link zur Creative Commons Lizenz beifügen und angeben, ob Änderungen vorgenommen wurden.

Die in diesem Kapitel enthaltenen Bilder und sonstiges Drittmaterial unterliegen ebenfalls der genannten Creative Commons Lizenz, sofern sich aus der Abbildungslegende nichts anderes ergibt. Sofern das betreffende Material nicht unter der genannten Creative Commons Lizenz steht und die betreffende Handlung nicht nach gesetzlichen Vorschriften erlaubt ist, ist für die oben aufgeführten Weiterverwendungen des Materials die Einwilligung des jeweiligen Rechteinhabers einzuholen. 